
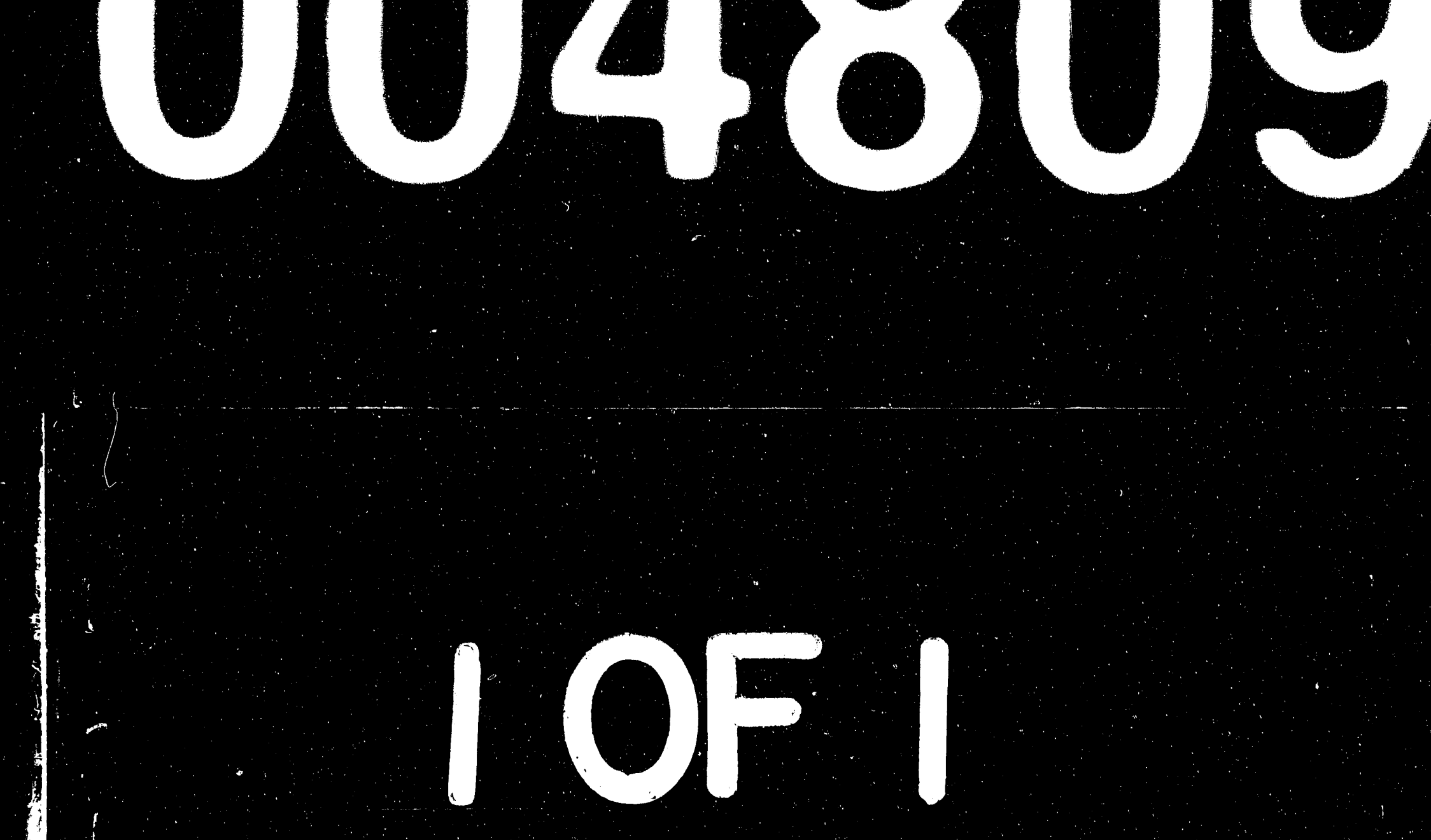

$\Gamma$
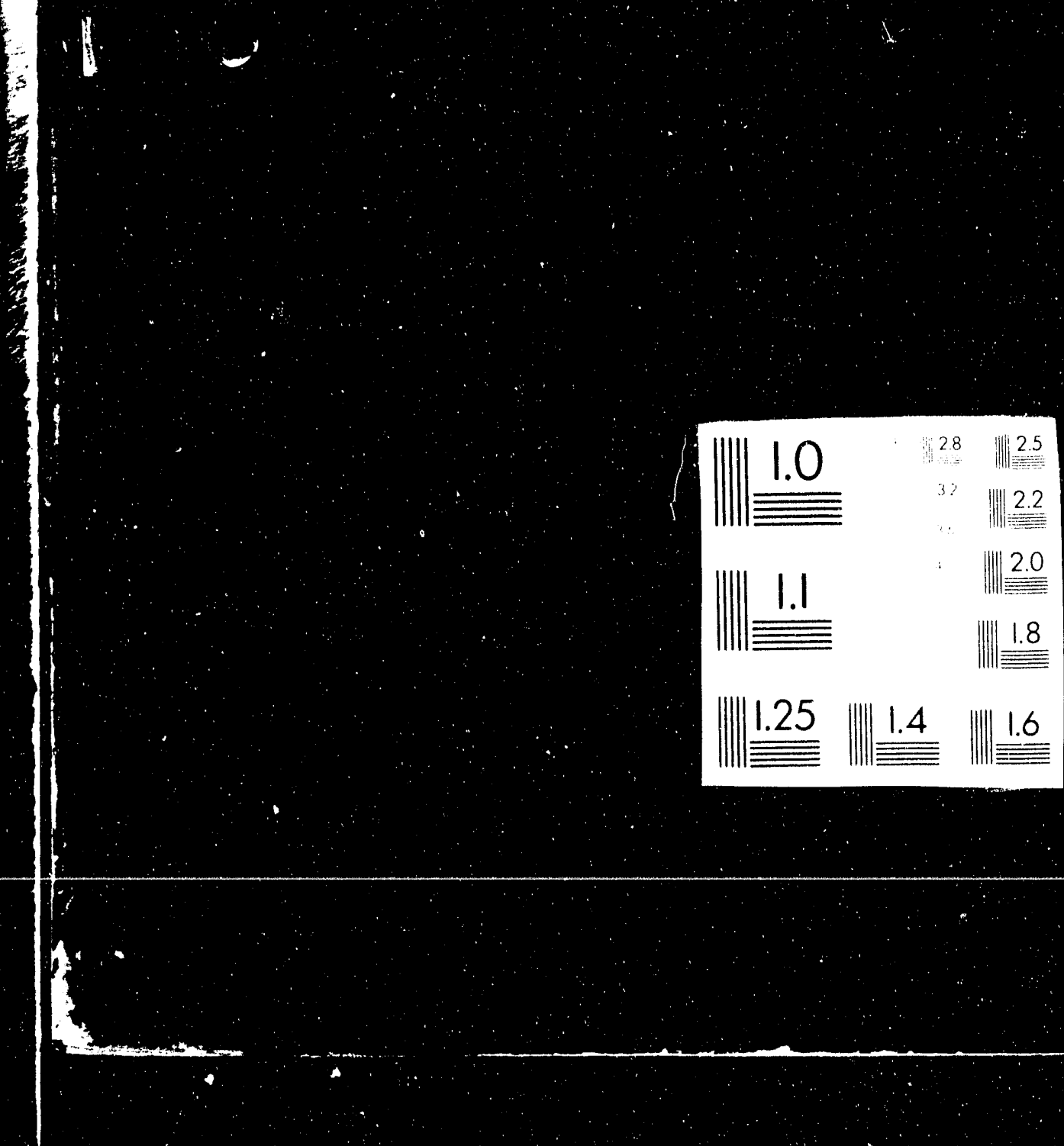


\title{
ENERGY DEPENDENCE OF AMORPHIZATION OF Ge BY Kr IONS*
}

\author{
R. C. Birtcher \\ Materials Science Division \\ Argonne National Laboratory \\ Argonne, IL 60439
}

$\mathrm{ANE} / \mathrm{MSD} / \mathrm{CP}-76483$

DE93 004809

\begin{abstract}
The submitted manuscript has been authored by a contractor of the U.S. Government under contract No. W-31-109-ENG-38. Accordingly, the U.S. Government retaine a nonexclustive, royalty-tree license to publish or reproduce the published lorm of this contribution, of alow others to do so, for U.S. Government purposes.
\end{abstract}

\section{DISCLAIMER}

This report was prepared as an account of work sponsored by an agency of the United States Government. Neither the United States Government nor any agency thereof, nor any of their employees, makes any warranty, express or implied, or assumes any legal liability or responsibility for the accuracy, completeness, or usefulness of any information, apparatus, product, or process disclosed, or represents that its use would not infringe privately owned rights. Reference herein to any specific commercial product, process, or service by trade name, trademark, manufacturer, or otherwise does not necessarily constitute or imply its endorsement, recommendation, or favoring by the United States Government or any agency thereof. The views and opinions of authors expressed herein do not necessarily state or reflect those of the United States Government or any agency thereof.

Submitted to the 1992 Materials Research Socieiy Fall Meeting, November 30December 4, 1992, Boston, MA.

*Work supported by the U. S. Department of Energy, BES-Materials Sciences, under Contract W-31-109-Eng-38.

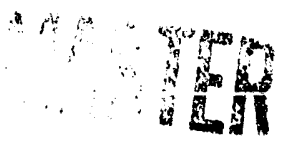


R. C. Birtcher

Materials Science Division, Argonne National Laboratory, Argonne, IL 60439

\section{ABSTRACT}

Thin Ge specimens have been irradiated with $\mathrm{Kr}$ ions of different energies, and the dose required for complete amorphization determined by in situ transmission electron microscopy. Because $\mathrm{Ge}$ is directly amorphized by a single energetic $\mathrm{Kr}$ ion, onset of amorphization was detected after the lowest ion doses. The $\mathrm{Kr}$ dose required for complete amorphization was found to increase linearly with ion energy over the range $0.5 \mathrm{MeV}$ to $3.5 \mathrm{MeV}$. With the assumption that the defect density required for amorphization is independent of ion energy, the number of defects produced in a thin specimen by each ion decreases with increasing energy as the reciprocal of the incident ion energy. TRIM calculations indicate that there is a slight decrease in the amount of damage required with increasing ion energy.

\section{INTRODUCTION}

The use of ion beam irradiation to alter surface layers by chemical or structural modification has become wide spread. One such surface modification is ion beam amorphization. Understanding amorphization of materials by energetic ions requires a knowledge of the interaction between an ion and the material and the relationship between defects and the transformation to the amorphous phase. Complete understanding of ion beam amorphization of materials in which ions are stopped requires determination of the energy or depth dependence of amorphization. In this work, the energy dependence of amorphization of $\mathrm{Ge}$ by $\mathrm{Kr}$ ions has been studied. This measurement allows calculation of the rate of amorphization in thick material as a function of depth from the surface. In part, the choice of Ge was motivated by the desire to use a mono-atomic system where ion energy loss is important and by observation of anomalous effects during ion beam modification of coated $\mathrm{Ge}$ surfaces [1]. In those experiments Ge coated with $\mathrm{Al}$ was irradiated with $120 \mathrm{keV}$ In ions at room temperature. Ion beam mixing was superseded by the growth of columnar voids extending into the $\mathrm{Ge}$ many times deeper than the calculated ion range. This effect was later identified as due to plastic flow of amorphous Ge during irradiation [2,3].

\section{EXPERIMENTAL DETAILS}

This in-situ study was performed at the HVEM-Tandem Accelerator Facility [4] at Argonne National Laboratory. The facility consists of a modified Kratos/AE1 EM7 high voltage electron microscope (HVEM) and a 2 
MV tandem National Electrostatics ion accelerator. The samples were jetpolished [5] polycrystalline Ge (99.99999 at. \% pure) TEM discs with grain size $>5 \mu \mathrm{m}$ in dimension. The only defects observed in the as-polished samples were isolated dislocations and grain boundaries. Irradiations were performed out at room temperature, and a separate specimen was used for each ion energy. Specimen thicknesses in the observed areas were determined by electron energy loss techniques in a Philips 420 electron microscope to be about $500 \AA$. The highest temperature produced by beam heating during ion irradiation was less than $48{ }^{\circ} \mathrm{C}$. The electron energy in the HVEM was maintained at $300 \mathrm{kV}$ which is below the displacement threshold of $\mathrm{Ge}$ for electron irradiation [6].

The amorphization dose was determined for $\mathrm{Kr}$ energies between 0.5 and $3.5 \mathrm{MeV}$. A dose rate of about $3.4 \cdot 10^{11} \mathrm{Kr} / \mathrm{cm}^{2} \mathrm{sec}$ was used for all irradiations to permit accurate determination of the amorphization dose. The amorphization doses are uncertain by approximately $2 \cdot 10^{12} \mathrm{Kr} / \mathrm{cm}^{2}$. In order to avoid an increase in the amorphization dose because of synergistic effects from the electron beam [3], the electron beam was turned off during ion irradiations. Periodically during each irradiation, the ion beam was interrupted and electron diffraction patterns were recorded. The amorphization dose was determined from the first electron diffraction micrograph that did not contain Bragg diffraction spots from crystalline Ge.

Because of direct amorphization of Ge by a single ion [7], a diffuse ring was detectable in the diffraction pattern after a very low $\mathrm{Kr}^{+}$dose. This appeared to be limited only by detectability on the microscope viewing screen of a weak diffuse ring in the presence of intense diffraction spots. For example at $1.5 \mathrm{MeV}$, a diffuse ring was observed in the diffraction pattern after doses as low as $3.10^{11} \mathrm{Kr} / \mathrm{cm}^{2}$ or an estimated $0.01 \mathrm{dpa}$. A similar detectability limit restricted our ability to determine the ion dose for complete amorphization at which all Bragg diffraction spots had disappeared from a pattern containing several intense diffuse rings. For this reason, the amorphization dose was determined after examination of diffraction micrographs.

TRIM 91 [8] calculations, using $25 \mathrm{eV}$ as the target displacement energy: were performed for all $\mathrm{Kr}$ ion energies with a specimen thickness of $50 \mathrm{~nm}$. Calculations yield an average ion range of $0.3 \mu \mathrm{m}$ for $0.15 \mathrm{MeV} \mathrm{Kr}+$ ions in $\mathrm{Ge}$, and $2 \mu \mathrm{m}$ for $3.5 \mathrm{MeV} \mathrm{Kr}$ ions. At $0.15 \mathrm{MeV}$, about $35 \%$ of the $\mathrm{Kr}$ ions are retained within the electron transparent region of the TEM specimens while the average ion energy loss through the specimen was about $70 \%$ of the initial energy. These problems do not arise at slightly higher energies. At 0.5 $\mathrm{MeV}$, the retained fraction of $\mathrm{Kr}$ was less than $2 \%$, and the amorphization dose of $0.8 \cdot 10^{14} \mathrm{Kr} / \mathrm{cm}^{2}$ resulted in an average implanted $\mathrm{Kr}$ concentration of less than 7 appm in the electron transparent region of the specimen. At higher energies, even less $\mathrm{Kr}$ is retained in the specimen in the electron transparent areas of the Ge sample. The calculations indicate that sputtering is less than $0.1 \mathrm{~nm}$ after any amorphization dose. TRIM calculation show a decrease in damage production within $100 \AA$ of both specimen surfaces for all ion energies used. It is not possible at this time to determine the impact of such damage gradients on the amorphization process, doses measured or 
average displacements calculated. For this reason, atom displacements averaged over the specimen thicknesses have been used.

\section{RESULTS}

The transformation from the crystalline to the amorphous state at room temperature is illustrated by a series of electron diffraction patterns taken during interruptions of a $3.5 \mathrm{MeV} \mathrm{Kr}$ irradiation, Fig. 1. The first sign of amorphization, evident by the appearance of a weak ring in the electron diffraction pattern, was detectable by electron diffraction after $3.5 \cdot 10^{12}$ $\mathrm{Kr} / \mathrm{cm}^{2}$. This represents an upper estimate of amorphization onset due to the difficulty in detecting a weak intensity that is spread out in a diffuse ring in the presence of a strong intensity localized in a spot pattern. Shown in Fig. $1(\mathrm{a})$, (b) and (c) are electron diffraction patterns from a Ge specimen before and after irradiating with $3.5 \mathrm{MeV} \mathrm{Kr}$ ion to doses of 1.71014 and $2.0 \cdot 10^{14}$ $\mathrm{Kr} / \mathrm{cm}^{2}$ respectively. Diffraction from crystalline $\mathrm{Ge}$ was still visible on micrographs after a dose of $1.9 \cdot 10^{14} \mathrm{Kr} / \mathrm{cm}^{2}$.

${ }^{\circ} \mathrm{A}$

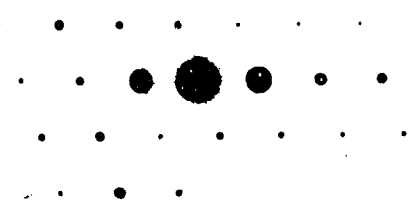

B

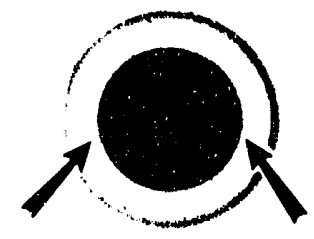

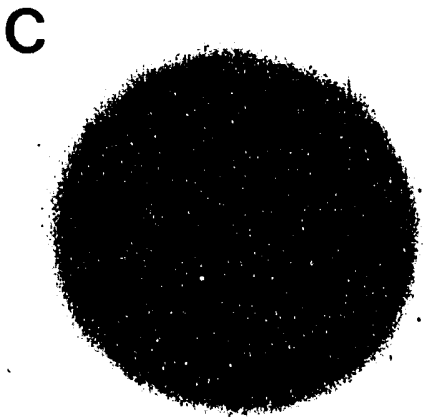

Figure 1 Electron diffraction patterns from Ge before irradiation (A) and after irradiation with $3.5 \mathrm{MeV} \mathrm{Kr}$ ions to dose of $1.710^{14}$ (B) and $2.0 \cdot 10^{14} \mathrm{Kr} / \mathrm{cm}^{2}(\mathrm{C})$.

The specimen remained in a mixed crystalline and amorphous state, as evidenced by the combination of both rings and spots, until complete amorphization at a dose of $2.0 \cdot 10^{14} \mathrm{Kr} / \mathrm{cm}^{2}$ when diffraction spots were no longer detectable on the micrographs. As with amorphization onset, the ion dose for full amorphization is affected by the experimentalist's ability to detect diffraction from a crystal lattice in the presence of much more intense diffuse scattering from amorphous material. We believe that these doses are uncertain by less than $5 \%$.

Similar measurements were performed for ion energies between $0.15 \mathrm{MeV}$ and 3.5 MeV. Separate specimens were used for each ion energy. The energy dependence of the $\mathrm{Kr}$ ion dose required to fully amorphize $\mathrm{Ge}$ is shown in Figure 2. Because of ion energy loss in passing through the specimen, 


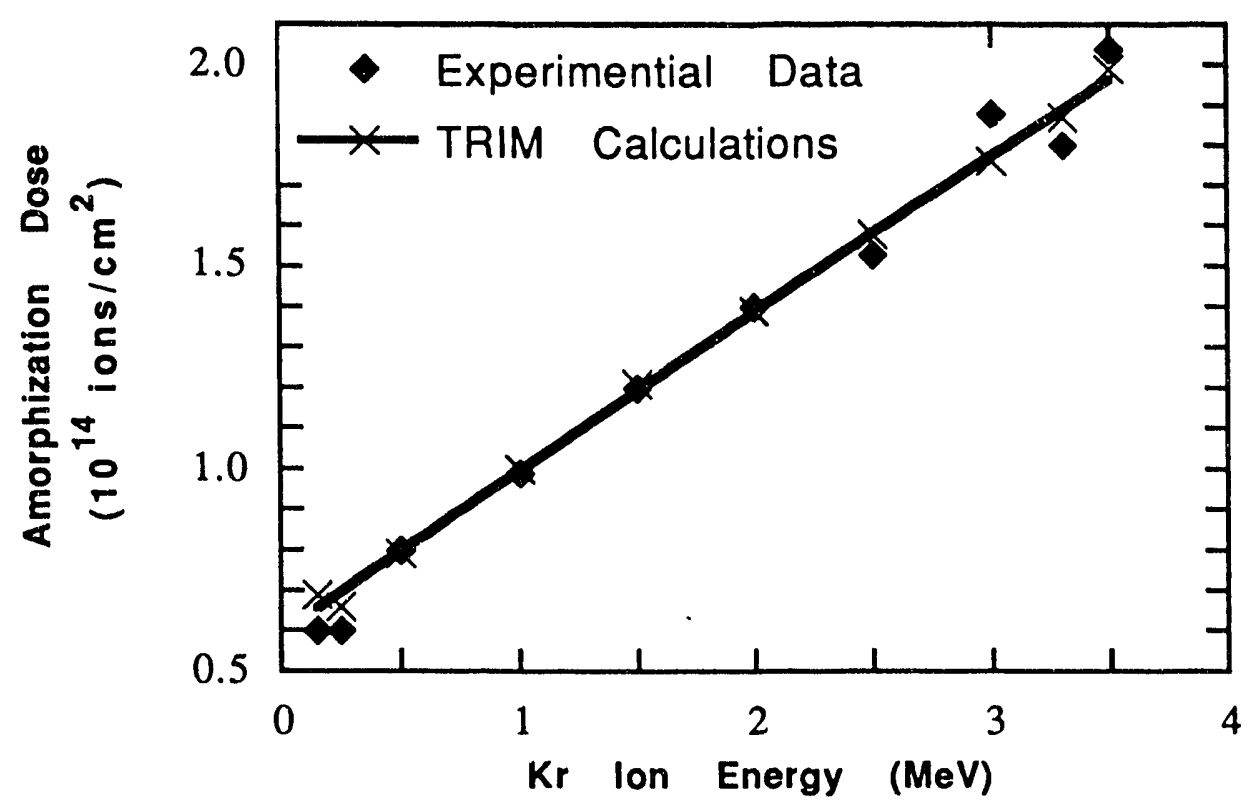

Figure 2 Ion dose required for complete amorphization of Ge.

the results reflect the average damage production by $\mathrm{Kr}$ ions at energies slightly below those used for each irradiation. With increasing ion energy, the data show a monotonic increase in the ion dose required for full amorphization and is adequately described by as linear function of ion energy. The two points at the lowest energies are more strongly affected by ion energy loss and stopping in the thin specimens.

The increase in required ion dose arises from a decrease in the collision cross section with increasing ion energy. Full TRIM 91 cascade calculations were made for each ion energy incident on $500 \AA$ thick Ge, and the average amount of damage was determined. This allows calculation of the number of displacements per atom (dpa) required for amorphization as a function of energy. The results are shown in Figure 3. The dpa required to fully amorphize $\mathrm{Ge}$ decreases with increasing $\mathrm{Kr}$ energy from 0.29 at our lowest energy to a value of 0.27 at an energy of $3.5 \mathrm{MeV}$. The least square fit has a slope of $-0.0057 \mathrm{dpa} / \mathrm{MeV}$. All calculated dpa's are sensitive to cascade termination at both surfaces. The values of the calculated amorphization dpa dose at our lowest energies are sensitive to ion stopping in the specimen. For example, TRIM calculations estimate that the fraction of $\mathrm{Kr}$ ion stopped is $35 \%$ at $0.15 \mathrm{MeV}, 12 \%$ at $0.25 \mathrm{MeV}, 2 \%$ at $0.5 \mathrm{MeV}$ and much less at higher energies. Since the dpa results are sensitive to the details of the TRIM calculation, they should not be taken to seriously. 


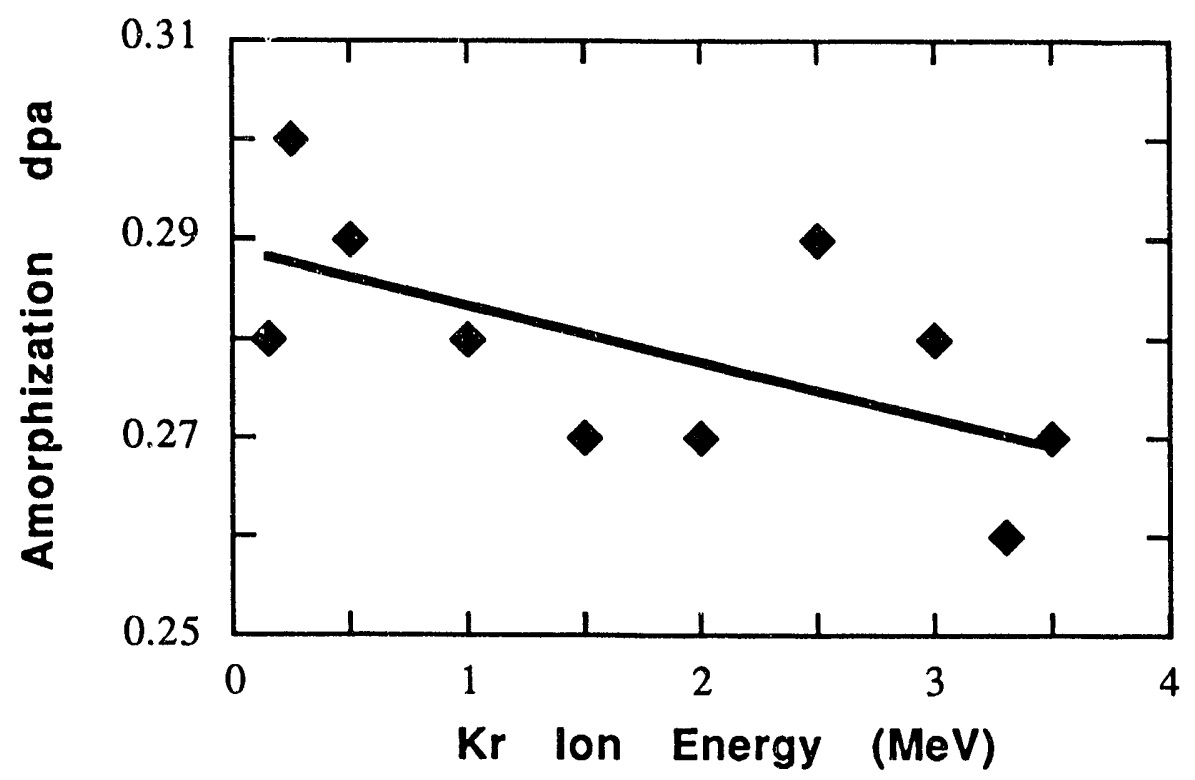

Figure 3 Displacements per atom required for complete amorphization of Ge.

Although TRIM does a good job of describing overall features of ion range and energy loss, it contains several approximations that prohibit it from fully describing high energy displacement events found in this experiment. When viewing individual ion tracks in TRIM, it becomes apparent that the statistical nature of the collision process plays a very important role in damage production and thus amorphization. At energies above $1 \mathrm{MeV}$, most $\mathrm{Kr}$ ions penetrate the specimen producing only a few defects. However, an occasional ion will initiate high energy defect cascade that may extend over the thickness of the specimen. The cross section for cascade production depends on both ion energy and energy loss over the specimen thickness. A version of TRIM 90 was modified to determine the primary knock-on (PKA) energy distribution. Cascade production varies approximately as $1 / \mathrm{E}$. Each $0.7 \mathrm{MeV}$ ion will produce one or more $10 \mathrm{keV}$ defect cascades in our $500 \AA$ thick specimens while a $3.5 \mathrm{MeV} \mathrm{Kr}$ ion has a probability of 0.3 to produce such a cascade.

The primary knock-on energy distributions can be used to estimate the ion doses required for amorphization. We assumed that amorphization requires a critical defect density, and that each PKA above $0.5 \mathrm{keV}$ will amorphize a volume of the specimen proportional to its initial energy. No consideration was given to the total number of defects actually produced within a cascade or the location of a cascade within the specimen thickness. The total volume amorphized per ion at each ion energy was calculated by integration over the TRIM PKA energy spectra, and the amorphization dose is taken as proportional to the reciprocal of this volume. The value of the critical defect concentration required for amorphization was found to be 0.16 by normalization at an ion energy of $1.0 \mathrm{MeV}$. The results of these calculations are shown in Figure 2 along with the experimental data. The agreement is well within experimental and statistical uncertainties. A more detailed 
description of the calculation including additional types of ions will be published.

The ability of atomic displacement calculations to describe amorphization of Ge suggest that the electronic energy loss does not play a significant role. The ratio of ionization energy to damage energy increases rapidly with increasing ion energy in our energy range. The ionization energy increases from $500 \mathrm{ev} / \AA$ at $0.5 \mathrm{MeV}$ to $5800 \mathrm{ev} / \AA$ at $3.5 \mathrm{MeV}$ while the damage energy increases from $16 \mathrm{ev} / \AA$ to $39 \mathrm{ev} / \AA$. The importance of electron excitation on damage accumulation in $\mathrm{Ge}$ is well known. We have observed that the combination of $300 \mathrm{keV}$ electrons with a $1.5 \mathrm{MeV} \mathrm{Kr}$ ion beam increases the ion dose for full amorphization by more than 10\% [3]. Higher energy electrons have an even greater affect. This increase is likely due to increased defect annihilation either from subthreshold collision events or electronic stimulated defect motion. If ionization during ion irradiation plays an important role during ion beam amorphization, it must only affect cascade formation and not other parts of the ion track.

\section{ACKNOWLEDGEMENTS}

*Work supported by the U. S. Department of Energy, BES-Materials Sciences, under Contract W-31-109-Eng-38.

\section{$\underline{\text { References }}$}

1. B. R. Appleton, O. W. Holland, D. B. Poker, J Narayan and D. Fathy, Nucl. Instrum. Methods B7/8, 639 (1985).

2. L. M. Wang and R. C. Birtcher, Appl. Phys. Lett. 55 , 2494, (1989).

3. L. M. Wang and R. C. Birtcher, Phil. Mag. A, 1209, (1991).

4. A. Taylor, C. W. Allen and E. A. Ryan, Nucl. Instrum. Methods B24/25, 598 (1987).

5. B. J. Kestel, Ultramicroscopy $\underline{9}, 379$ (1982).

6. J. W. Corbett, Electron Radiation Damage in Semiconductors and Metals (Academic, New York, 1966), p. 134.

7. L. M. Howe and M. H. Rainville, Nucl. Instrum. Methods B19/20, 61 (1987).

8. J. Biersack and L. G. Haggmark, Nucl. Instr. and Meth. 174, 257 (1980). 

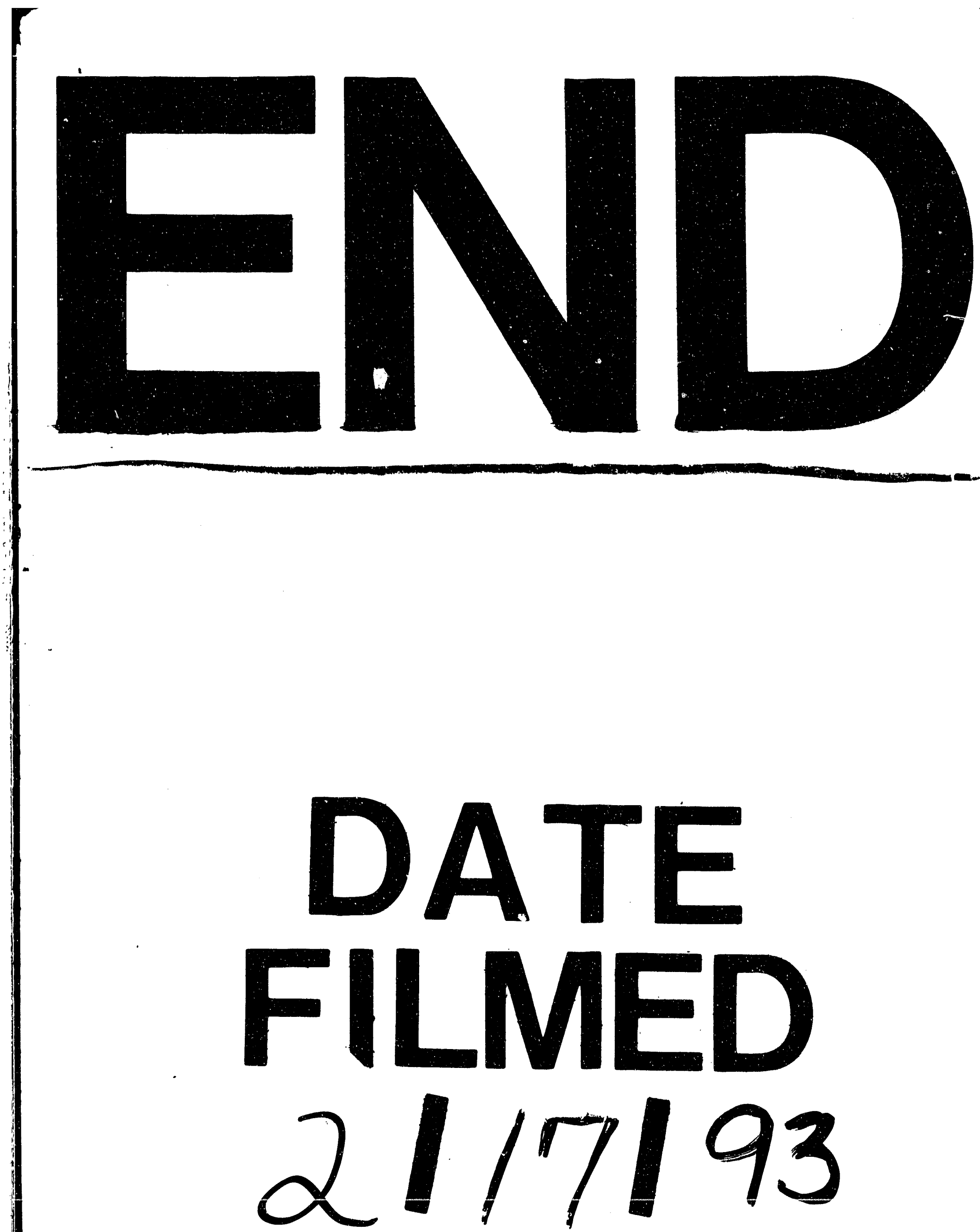
$\mid$ 